\title{
High-Performance Flexible Schottky Diodes Based on Sputtered InGaZnO
}

DOI:

10.1109/TED.2018.2864165

\section{Document Version}

Accepted author manuscript

Link to publication record in Manchester Research Explorer

\section{Citation for published version (APA):}

Du, L., Zhang, J., Li, Y., Xu, M., Wang, Q., Song, A., \& Xin, Q. (2018). High-Performance Flexible Schottky Diodes Based on Sputtered InGaZnO. IEEE Transactions on Electron Devices, 65(10), 4326-4333.

https://doi.org/10.1109/TED.2018.2864165

\section{Published in:}

IEEE Transactions on Electron Devices

\section{Citing this paper}

Please note that where the full-text provided on Manchester Research Explorer is the Author Accepted Manuscript or Proof version this may differ from the final Published version. If citing, it is advised that you check and use the publisher's definitive version.

\section{General rights}

Copyright and moral rights for the publications made accessible in the Research Explorer are retained by the authors and/or other copyright owners and it is a condition of accessing publications that users recognise and abide by the legal requirements associated with these rights.

\section{Takedown policy}

If you believe that this document breaches copyright please refer to the University of Manchester's Takedown Procedures [http://man.ac.uk/04Y6Bo] or contact uml.scholarlycommunications@manchester.ac.uk providing relevant details, so we can investigate your claim.

\section{OPEN ACCESS}




\title{
High-Performance Flexible Schottky Diodes Based on Sputtered InGaZnO
}

\author{
Lulu Du, Jiawei Zhang, Yunpeng Li, Mingsheng Xu, Qingpu Wang, Aimin Song, senior Member, \\ IEEE, and Qian Xin
}

\begin{abstract}
High-performance flexible InGaZnO (IGZO)-based Schottky diodes are fabricated on polyethylene-terephthalate (PET) and polyimide (PI) substrates at room temperature without any thermal treatment. The diode performance improves significantly by either oxygen plasma or UV-ozone treatment on Pd anode. X-ray photoelectron spectroscopy indicates that the both treatments lead to Pd surface oxidation. The oxygen plasma treatment results in more complete oxidation, and thus ensures better oxygen stoichiometry at the Schottky interface and a higher anode work function. This leads to high performance with on/off ratios of $7.3 \times 10^{6}$ and $2.6 \times 10^{4}$, barrier heights of 0.79 and 0.76 $\mathrm{eV}$, and ideality factors of 1.22 and 1.19 on PET and PI, respectively. Interestingly, these flexible diodes show improved performance after storing in ambient air for two years, and fittings on the reverse currents indicate an improved barrier uniformity. The flexible diode on PET after a two-year storage achieves a high on/off ratio of $2.0 \times 10^{7}$, a large barrier height of $0.80 \mathrm{eV}$, a close to unity ideality factor of 1.09 , a high breakdown voltage of $-7.5 \mathrm{~V}$, and robust performance upon outward or inward bending, which is, to the best of our knowledge, the highest performance in reported flexible diodes to date.
\end{abstract}

Index Terms-InGaZnO (IGZO), Schottky diode, flexible, oxidation treatment.

\section{INTRODUCTION}

$\mathrm{S}$ EMICONDUCTOR devices and circuits on flexible substrates are highly desirable in applications such as flexible displays, lighting, radio-frequency identification tags, electronic skins, and wearable electronics [1]-[3]. Oxide semiconductors are considered as one of the most suitable candidates for flexible electronics because of their high carrier mobility $\left(\sim 1-100 \mathrm{~cm}^{2} / \mathrm{Vs}\right)$ compared to amorphous silicon and organic semiconductors, large-area and low-temperature processibility, and good transparency in the visible light region [4]-[6]. To date, flexible oxide semiconductor thin-film transistors, one of the essential elements for flexible circuits, have been extensively studied and even started to be commercialized as the backplane driver of displays [7], [8]. Flexible diodes based on oxide semiconductors [9]-[16], on the

Manuscript received Mar. 29, 2018. This work was supported by the National Key Research and Development Program of China (Grant No. 2016YFA0301200 and 2016 YFA0201800), Engineering and Physical Sciences Research Council (EPSRC) (Grant No. EP/N021258/1), the Natural Science Foundation of Shandong Province (ZR201709260014), the Key Research and Development Program of Shandong Province (2017GGX10111 and 2017GGX10121), China Postdoctoral Science Foundation funded project other hand, have not been paid much attention despite being another fundamental block in various circuits such as logic gates, resistive random-access memory, radio-frequency identification tags, etc. [17]-[19]. Overall, two major challenges remain for flexible Schottky diodes, namely large ideality factors and high reverse currents both resulting in low rectification ratios and low breakdown voltages. Various efforts have been made to improve the quality of oxide semiconductor Schottky contacts. It was reported that annealing in oxygen gas at $200{ }^{\circ} \mathrm{C}$ could increase the rectification ratio of IGZO Schottky diodes due to the reduction of interface states density [9], [20]-[22]. However, such annealing treatment is not suitable for many plastic substrates with a low glass transition temperature, e.g., polyethylene-terephthalate (PET). Annealing may also introduce stress and interface trap states at the heterojunctions and thus result in degradation of the device performance [23], [24]. Another method to avoid oxygen vacancies at the Schottky interface is by deposition of oxide layers [16], [25], [26] or applying oxidation treatment on the Schottky anode [22], [24]. Introducing sputtered oxide layers such as Ru-Si-O [16], $\mathrm{AgO}_{\mathrm{x}}$ [25] or $\mathrm{PtO}_{\mathrm{x}}$ [26], at IGZO Schottky interface have successfully achieved large Schottky barrier heights $\left(\Phi_{\mathrm{B}}\right)$ of $0.90 \sim 1.00 \mathrm{eV}$. However, it leads to relatively large ideality factors $(n)$ of $1.32 \sim 1.97$, probably due to the interface defects induced by ion bombardment during the sputtering deposition. Oxygen plasma or UV-ozone treatment on some Schottky anode metals ( $\mathrm{Pd}, \mathrm{Au})$ prior to the deposition of oxide semiconductor has been reported to enhance the interface oxygen stoichiometry and Schottky barrier height, however, annealing is commonly required to achieve high performance [22], [24], [27].

In this work, by a combination of efficient oxygen plasma treatment on Pd anode and IGZO sputtering with low oxygen partial pressure, high performance flexible diodes were achieved without annealing. These flexible diodes have achieved $n$ of 1.22 and $1.19, \Phi_{\mathrm{B}}$ of 0.79 and $0.76 \mathrm{eV}$, and high on/off ratio $\left(I_{\text {on/off }}\right)$ of $7.3 \times 10^{6}$ and $2.6 \times 10^{4}$ at $\pm 1 \mathrm{~V}$, on PET and PI, respectively. Interestingly, after storing in ambient air

(2016M590634), and the Fundamental Research Funds of Shandong University (2016WLJH44).

L. Du, Y. Li, M. Xu, Q. Wang, A. Song, Q. Xin are with the Centre of Nanoelectronics, State Key Laboratory of Crystal Materials, and School of Microelectronics, Shandong University, Jinan 250100, China (e-mail: xinq@sdu.edu.cn).

J. Zhang, A. Song are with the School of Electrical and Electronic Engineering, University of Manchester, Manchester M13 9PL, UK. 
for two years, these flexible diodes show not only no performance degradation but in the contrary significant improvement. The diodes on PET show the best performance with the lowest $n$ of 1.09 , highest $I_{\text {on/off }}$ of $2.0 \times 10^{7}$, and largest $\Phi_{\mathrm{B}}$ of $0.80 \mathrm{eV}$.

\section{EXPERIMENTAL SECTION}

\section{A. Rigid and Flexible Devices Fabrication}

Rigid $\mathrm{SiO}_{2} / \mathrm{Si}$, and flexible PET and PI were used as the substrates. $\mathrm{SiO}_{2} / \mathrm{Si}$ substrates were sequentially cleaned in an ultrasonic bath of decon, de-ionized water, acetone, and ethanol, and dried by $\mathrm{N}_{2}$. The cleaned $\mathrm{SiO}_{2} / \mathrm{Si}$ were then annealed at 300 ${ }^{\circ} \mathrm{C}$ for $30 \mathrm{~min}$ to remove moistures and organic adsorbate [28]. The PET and PI were ultrasonically cleaned in acetone and ethanol subsequently, and then dried by $\mathrm{N}_{2}$ without any thermal treatment. A 3-nm-thick Ti layer was deposited on the rigid or flexible substrates for better adhesion. A 47-nm-thick Pd layer was deposited as Schottky contact, and then treated by either UV-ozone (PDC $32 \mathrm{G}$ ) or oxygen plasma (ProCleanerTM). The oxygen plasma treatment was performed at a power of $100 \mathrm{~W}$ and a radio-frequency of $13.56 \mathrm{MHz}$. A 100-nm-thick IGZO layer was deposited by RF magnetron sputtering at room temperature with an $\mathrm{InGaZnO}_{4}$ ceramic target with a $\mathrm{RF}$ sputtering power of $70 \mathrm{~W}$ and an oxygen partial pressure [oxygen/(oxygen + argon)] of $2.5 \%$. Finally, $\mathrm{Ti}(40 \mathrm{~nm}) / \mathrm{Au}(40$ $\mathrm{nm}$ ) was deposited as the ohmic contact through a shadow mask. All the metals were deposited by electron-beam evaporation. All the diodes have an active area of $9.5 \times 10^{-4} \mathrm{~cm}^{2}$. The cross section structure of the rigid diodes is shown in the inset of Fig. 1(a). The flexible diodes have the same device structure as the rigid diodes.

\section{B. Device Characterization}

The electrical characteristics of these diodes were analyzed by current density-voltage $(J-V)$ and capacity-voltage $(C-V)$ measurements using a source measure unit (Agilent 2902B) and an LCR meter (Agilent E4980A, $1 \mathrm{MHz}$ ), respectively, at room temperature. The composition of Pd surface was determined by X-ray photoelectron spectroscopy (XPS, ESCALAB 250). The surface roughness was analyzed by atomic force microscope (AFM, Dimension FastScan ${ }^{\mathrm{TM}}$ ).

\section{RESULTS AND DISCUSSIONS}

\section{A. Effect of oxidation treatments on the diode performance}

It is reported that high Schottky barrier can be achieved by sputtering IGZO layers with relatively high oxygen partial pressure of $20 \%$ to ensure high oxygen stoichiometry in the Schottky interface [22], [24]. However, such high oxygen partial pressure can induce high interface trap density [29] and degrade the diode performance (e.g. large $n$, large barrier inhomogeneity, etc.). Therefore, annealing is commonly required to reduce interface trap density [22], [24]. In this work, by applying efficient oxygen plasma treatment on $\mathrm{Pd}$ anode and deposition of IGZO with low oxygen partial pressure of $2.5 \%$, high performance IGZO diodes with low interface trap density were achieved without annealing, as we discuss infra.
IGZO/Pd diodes on $\mathrm{SiO}_{2} / \mathrm{Si}$ are fabricated to study the effects of oxidation treatments on device performance. Fig. 1(a)-(c) show the $J-V$ curves of the diodes, which can be analyzed by a thermionic emission model given by [30],

$$
\begin{aligned}
& J=J_{S}\left\{\exp \left[\frac{q\left(V-I R_{S}\right)}{n k T}\right]-1\right\} \\
& J_{S}=A^{*} T^{2} \exp \left(\frac{-\Phi_{B, J V}}{k T}\right)
\end{aligned}
$$

where $J_{\mathrm{S}}$ is the saturation current density, $A^{*}$ is the effective Richardson constant which is $41 \mathrm{Acm}^{-2} \mathrm{~K}^{-2}$ for IGZO [10], [31], $T$ is the temperature, $q$ is the elementary charge, $\Phi_{\mathrm{B}, J V}$ is the effective barrier height determined by $J-V$ characteristics, $k$ is the Boltzmann constant, and $R_{\mathrm{S}}$ is the series resistance.
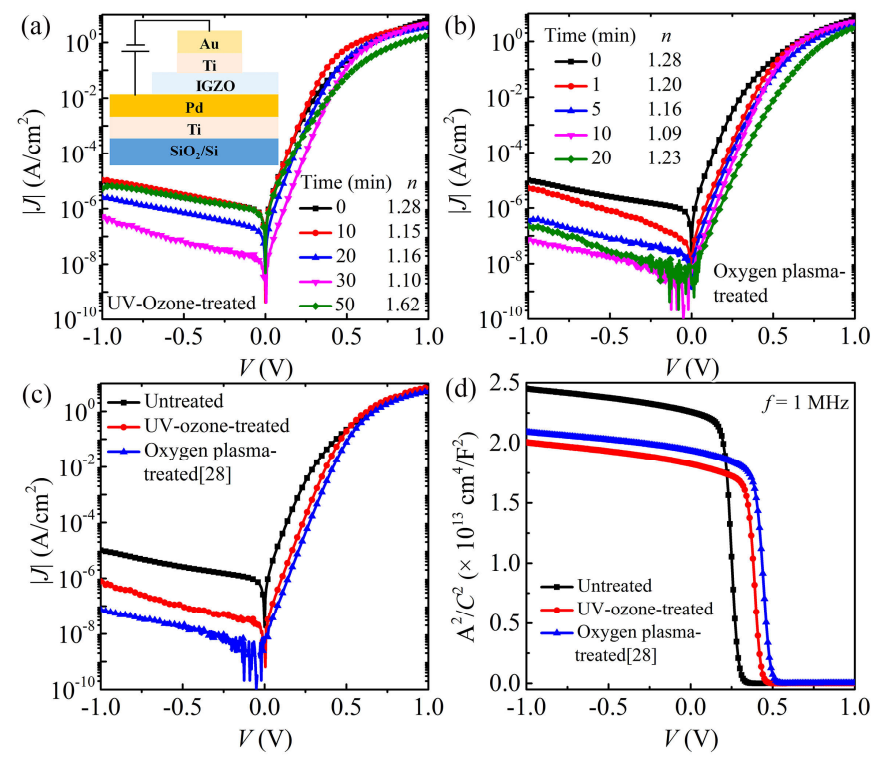

Fig. 1. $J-V$ characteristics of the diode with UV-Ozone (a) or oxygen plasma (b) treatment on anode Pd at different time. $J-V(\mathrm{c})$ and $C-V(\mathrm{~d})$ characteristics of untreated, UV-ozone treated, and oxygen plasma treated IGZO/Pd diodes with the optimized parameters on $\mathrm{SiO}_{2} / \mathrm{Si}$ substrates. The optimal oxygen plasma treated data are reproduced with permission [28]. Inset shows the cross section of the diodes.

The optimized treatment time of oxygen plasma and UVozone treatments are studied, as shown in Fig. 1(a) and (b). It should be noticed that prolonged treatments than the optimized time (10 min for oxygen plasma treatment and $30 \mathrm{~min}$ for UVOzone treatment) led to no performance enhancement but degradation with increased $n$, as shown in Fig. 1(a) and (b), most probably due to increase of interface subgap trap density.

The $\Phi_{\mathrm{B}, J V}$ values are $0.73,0.84$ and $0.88 \mathrm{eV}$ for untreated, UV-ozone treated, and oxygen plasma treated diodes with the optimized parameters, respectively, as extracted from the $J-V$ curves by Equations (1) and (2). Notably, with oxidation treatment, the barrier height increases significantly, which results in an improvement of $I_{\mathrm{on} / \mathrm{off}}$ by one to two orders of magnitude. This is attributed to the chemical modification (oxidation) of $\mathrm{Pd}$ and the removal of surface contaminants such as hydrocarbons, which can restrain the oxygen deficiency in IGZO and increase the work function of Pd surface [24], [27], [32]. The improved interface is also evidenced by the near unity $n$ of $\sim 1.1$ for oxidation treated devices, in contrast to the $n$ of 1.28 for untreated device. On the other hand, at an applied 
voltage of $1 \mathrm{~V}$, the on-current density $\left(J_{\text {on }}\right)$ is found almost identical for the three diodes, corresponding to a similar resistance $R_{\mathrm{S}}$ of $45 \sim 64 \mathrm{~m} \Omega \mathrm{cm}^{2}$ extracted from the $\mathrm{d} V / \mathrm{d} J$ at 1 $\mathrm{V}$. This is due to that the IGZO layers in all three samples were deposited under the same condition, and thus it is expected that both the doping concentration of the IGZO and the ohmic contact resistance with $\mathrm{Ti}$ should be the same in all three devices. In contrast, the off-current density $\left(J_{\text {off }}\right)$ exhibits pronounced process dependence, as shown in Fig. 1(c). Oxidation treatments enhance the Schottky barrier height by $110 \sim 150 \mathrm{meV}$, which could partly explain the reduced $J_{\text {off. }}$ The oxygen plasma treated diode exhibits the highest $\Phi_{\mathrm{B}, J V}$ and $I_{\mathrm{on} / \text { off }}$ of $0.88 \mathrm{eV}$ and $7.2 \times 10^{7}$, respectively, as shown in Table I, indicating that the oxygen plasma treatment may produce more complete oxidation of $\mathrm{Pd}$ and better stoichiometry at the interface than UV-ozone treatment. This is, to the best of our knowledge, the highest $I_{\mathrm{on} / \text { off }}$ for rigid Schottky diodes in which IGZO is not thermally annealed.

After oxidation treatment, both $J_{\text {off }}$ and $n$ decrease substantially. In order to understand such behavior, $C-V$ measurements are performed, and the results are plotted in Fig. 1(d). The capacitance of Schottky junction is given by [30],

$\frac{A^{2}}{C^{2}}=\left(\frac{2}{q \varepsilon_{s} \varepsilon_{0} N_{\text {depl }}}\right)\left(V_{b i}-\frac{k T}{q}-V\right)$

where $A$ is the active area of the diode, $\varepsilon_{\mathrm{s}}$ is the static dielectric constant of the semiconductor, $\varepsilon_{0}$ is the dielectric constant of vacuum, $N_{\text {depl }}$ is the background doping density in the depletion region, and $V_{\mathrm{bi}}$ is the built-in potential. The $A^{2} / C^{2}-V$ curvesillustrate that oxidation treatments can effectively enlarge the $V_{\mathrm{bi}}$ of diodes. The $q V_{\mathrm{bi}}$ values extracted from Equation (3) are $0.29,0.43$, and $0.51 \mathrm{eV}$, for untreated, UVozone treated, and oxygen plasma treated samples, respectively. The barrier height can be evaluated by the $C-V$ measurements by $\Phi_{B, C V}=q V_{b i}+\left(E_{c}-E_{f}\right)$, where the last term describes the energy difference between the conduction band minimum and Fermi level. The $E_{\mathrm{c}}-E_{f}$ values can be evaluated by $E_{c}-E_{f}=k \operatorname{Tln}\left(N_{c} / N_{e}\right)$, where $N_{\mathrm{c}}$ is the conduction band density of states and is $5.2 \times 10^{18} \mathrm{~cm}^{-3}$ for IGZO, and $N_{\mathrm{e}}$ is the free charge concentration which can be estimated from $R_{\mathrm{S}}$ [31]. The $E_{\mathrm{c}}-E_{f}$ values are evaluated to be $0.21,0.22$ and $0.23 \mathrm{eV}$ for untreated, UV-ozone treated, and oxygen plasma treated diodes, respectively, corresponding to the $\Phi_{\mathrm{B}, C V}$ of $0.50,0.65$, and 0.74 $\mathrm{eV}$, respectively. $\Phi_{\mathrm{B}, J V}$ is the effective barrier height, and is rather sensitive to the barrier inhomogeneities. In contrast, $C-V$ measurements give the mean barrier height which is usually larger than $\Phi_{\mathrm{B}, J V}$ for single crystalline semiconductors with extremely low trap state densities [33], [34]. However, amorphous IGZO have much more traps which can cause an extra capacitance and thus reduced $V_{\mathrm{bi}}$ during the $C-V$ measurements, due to the response of traps to the applied frequencies [35]. Thus, $\Phi_{\mathrm{B}, C V}$ is smaller than $\Phi_{\mathrm{B}, J \mathrm{~V}}$ for these IGZO diodes, as well as in other reported IGZO Schottky diodes [9], [21], [22]. The $J-V$ and $C-V$ results demonstrate that both oxidation treatments enhance both the Schottky barrier height and the barrier uniformity, and the oxygen plasma treatment leads to a higher $\Phi_{\mathrm{B}}$ and better $n$ than the UV-zone treatment.
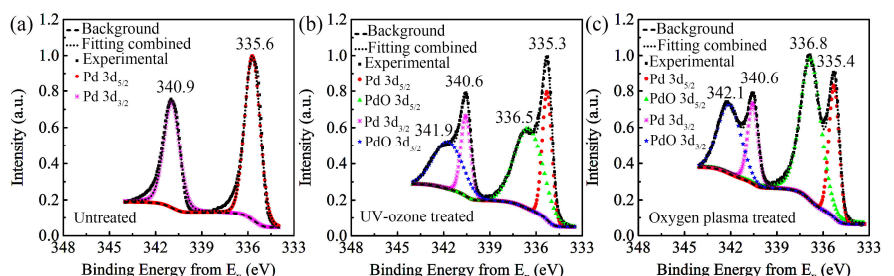

Fig. 2. XPS spectra of (a) untreated, (b) UV-ozone treated and (c) oxygen plasma treated Pd samples with fittings.

As the results show that oxygen plasma treated diode has better performance, we have investigated on the oxidation of $\mathrm{Pd}$ under oxygen plasma (10 $\mathrm{min}$ ) or UV-ozone (30 min) treatment by XPS. The untreated Pd shows only two Pd peaks: $3 \mathrm{~d}_{5 / 2}$ and $3 \mathrm{~d}_{3 / 2}$ centered at 335.6 and $340.9 \mathrm{eV}$ [24], [27], respectively, as shown in Fig. 2(a). The oxidation treated samples show clear evidence of Pd oxidation as the presence of $\mathrm{Pd}^{2+}$ peaks: $3 \mathrm{~d}_{5 / 2}$ and $3 \mathrm{~d}_{3 / 2}$ centered at $\sim 337$ and $\sim 342 \mathrm{eV}$, respectively, as shown in Fig. 2(b) and (c). The ratio of PdO extracted from the XPS peaks for the oxygen plasma treated sample is $63.6 \%$, while that for the UV-ozone treated sample is $54.3 \%$. The results hence demonstrate that $\mathrm{Pd}$ can be oxidized to $\mathrm{PdO}$ by both oxygen plasma and UV-ozone treatment, and the oxygen plasma treatment in this experiment results in more complete Pd oxidation. The XPS results are consistent with the $J-V$ characteristics of the diodes. The results thus suggest that the oxidation treatment improved oxygen stoichiometry at the IGZO/Pd interface and enhanced the work function of the Pd anode.

TABLE I

CHARACTERISTICS OF RIGID SCHOTTKY DIODES FROM IV AND CV MEASUREMENTS

\begin{tabular}{cccc}
\hline \hline Treatments & Untreated & UV-ozone treated Oxygen plasma treated \\
\hline$R_{\mathrm{S}}\left(\mathrm{m} \Omega \cdot \mathrm{cm}^{2}\right)$ & 45 & 51 & 64 \\
$n$ & 1.28 & 1.10 & 1.09 \\
$I_{\text {on/off }}$ & $8.0 \times 10^{5}$ & $9.4 \times 10^{6}$ & $7.2 \times 10^{7}$ \\
$\Phi_{B, J V}(\mathrm{eV})$ & 0.73 & 0.84 & 0.88 \\
$\Phi_{B, C V}(\mathrm{eV})$ & 0.50 & 0.65 & 0.74 \\
$q V_{\mathrm{bi}}(\mathrm{eV})$ & 0.29 & 0.43 & 0.51 \\
\hline \hline
\end{tabular}
[28].

${ }^{a}$ The optimal oxygen plasma treated data are reproduced with permission

\section{B. High performance flexible Schottky didoes}

Based on the optimized oxygen plasma treatment process parameters, high performance flexible IGZO diodes on PET and PI have been fabricated without any thermal treatment. The $J-V$ curves of the as-deposited diode on PET and the diode being kept in ambient air for 12 and 25 months are shown in Fig. 3(a), and the diode performance parameters are shown in Table II. The mean effective barrier height exhibits negligible changes. $J_{\text {on }}$ increases with the storage time. $I_{\text {on/off }}$ changed from $7.3 \times 10^{6}$ to $2.7 \times 10^{6}$ and $2.0 \times 10^{7}$, and $n$ improved from 1.22 to 1.12 and 1.09 after being kept in ambient air for 12 and 25 months, respectively. Fig. 3(b) shows the $J-V$ characteristics of the as-deposited diode on PI with the storage time. The asdeposited diode shows high reverse current which depends strongly on the applied bias, and this could be attributed to the image force lowering and barrier inhomogenities [36]. After 

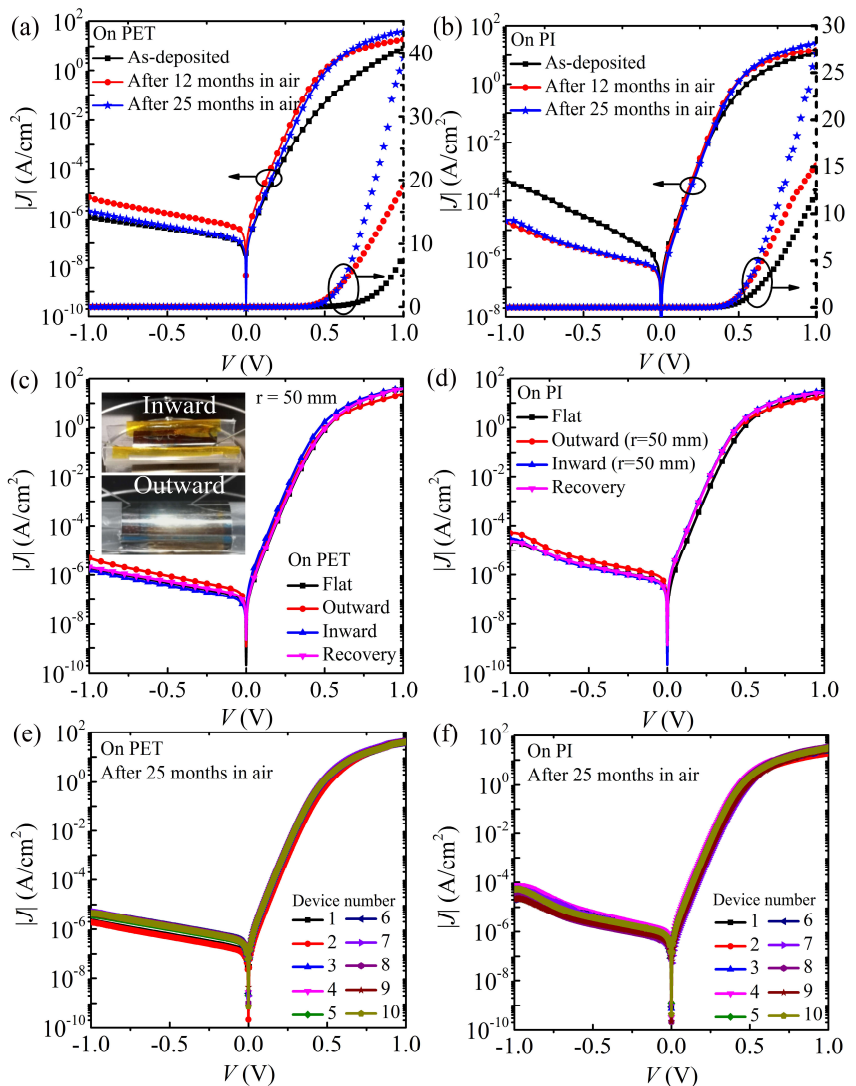

Fig. 3. Stability of the IGZO/Pd flexible Schottky diodes on PET (a) and PI (b). $J-V$ characteristics measured before bending, under outward or inward bending, and after removal of bending (recovery) for the diodes on PET (c) and PI (d). J-V curves uniformity of the diodes on PET (e) and PI (f). The inset of Fig. 3(c) is a photo showing the diodes attached to a plastic pipe for bending test.

leaving the diode on PI in ambient air for 12 and 25 months, the diode also exhibits appreciable improvement with better $n$, $\Phi_{\mathrm{B}, J V}$ and $I_{\text {on/off. }}$ The $J_{\text {on }}$ is slightly increased with the storage time, and the $J_{\text {off }}$ is decreased by one order of magnitude a year later and remained unchanged until two years later. The $n$ improves from 1.19 to 1.12 and 1.11, and the $I_{\mathrm{on} / \mathrm{off}}$ increased from $2.6 \times 10^{4}$ to $7.9 \times 10^{5}$ and $1.3 \times 10^{6}$, after being kept in ambient air for 12 and 25 months, respectively, indicating higher Schottky interface quality. The detailed parameters are shown in Table II. The improved performances are suggested to be due to the improved barrier homogeneity probably caused by the release of interface stresses. The results illustrate that these flexible IGZO-based Schottky diodes can hold or even improve the diode performance when they are exposed to ambient air for long time, providing positive implications for real applications. Compared with the rigid diode, the flexible diodes show slightly degraded performances, and this is mainly due to the flexible substrates have larger surface roughness, as revealed by AFM measurements and shown in Fig. 4. However, although the flexible diodes have rougher IGZO/Pd interfaces, they still show relatively high performances without any thermal treatment, because that the oxidation of $\mathrm{Pd}$ at the Schottky interface guarantees a relatively high $\Phi_{\mathrm{B}}$ and small $n$. To the best of our knowledge, the flexible diode on PET in this work exhibits the best overall performance with the largest $\Phi_{\mathrm{B}}$. ${ }_{J V}$ of $0.80 \mathrm{eV}$, highest $I_{\text {on/off }}$ of $2.0 \times 10^{7}$, which is $1 \sim 2$ orders higher than the reported maximum value, and lowest $n$ of 1.09, among the reported flexible Schottky diodes to date, as shown in Table III, and even better than many rigid IGZO Schottky diodes [24], [31].
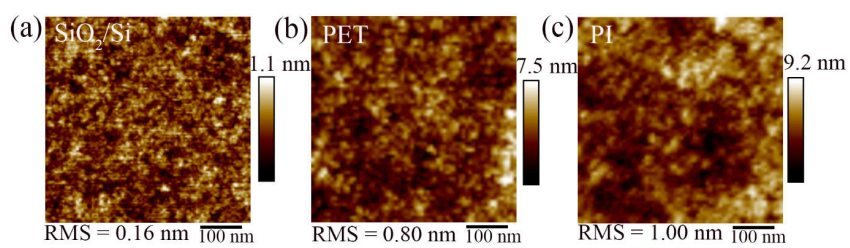

(d)
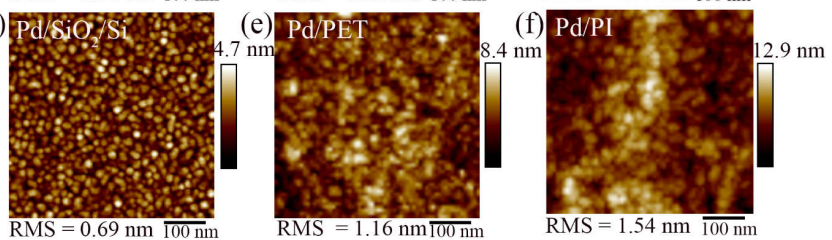

ig. 4. AFM images and RMS surface roughnesses of a) $\mathrm{SiO}_{2} / \mathrm{Si}$, b) PET, c) PI substrates, and Pd thin films on d) $\mathrm{SiO}_{2} / \mathrm{Si}$, e) PET, and f) PI substrates.

TABLE II

CHARACTERISTICS OF FLEXIBLE SCHOTTKY DIODES FROM IV MEASUREMENTS AND REVERSE CURRENT FITTINGS

\begin{tabular}{ccccccc}
\hline \hline & \multicolumn{2}{c}{ As-deposited } & \multicolumn{2}{c}{12 months } & \multicolumn{2}{c}{25 months } \\
\hline Substrates & PET & PI & PET & PI & PET & PI \\
\hline$R_{\mathrm{s}}\left(\mathrm{m} \Omega \mathrm{cm}^{2}\right)$ & 20 & 29 & 23 & 29 & 12 & 21 \\
$n$ & 1.22 & 1.19 & 1.12 & 1.12 & 1.09 & 1.11 \\
$I_{\text {on/off }}$ & $7.3 \times 10^{6}$ & $2.6 \times 10^{4}$ & $2.7 \times 10^{6}$ & $7.9 \times 10^{5}$ & $2.0 \times 10^{7}$ & $1.3 \times 10^{6}$ \\
$\Phi_{\mathrm{B}, V V}(\mathrm{ev})$ & 0.79 & 0.76 & 0.77 & 0.77 & 0.80 & 0.78 \\
$\Phi_{B}(\mathrm{ev})$ & 0.85 & 0.80 & 0.83 & 0.82 & 0.87 & 0.82 \\
$N_{\text {depl }}\left(\times 10^{17} \mathrm{~cm}^{-3}\right)$ & 5.13 & 5.85 & 9.20 & 6.45 & 12.0 & 8.25 \\
$\gamma\left(\times 10^{-4} \mathrm{~cm}^{2 / 3} \mathrm{~V}^{1 / 3}\right)$ & 1.46 & 1.86 & 1.28 & 1.46 & 1.11 & 1.37 \\
$r(\mathrm{~nm})$ & 15 & 22 & 12 & 15 & 10 & 14 \\
\hline \hline
\end{tabular}

Schottky barrier height $\left(\Phi_{\mathrm{B}}\right)$, background doping density $\left(N_{\text {depl }}\right)$, region parameter $(\gamma)$, and effective radius of low barrier height $(r)$ by simulation.

To evaluate the susceptibility of the diode performance to bending, the diodes were outward and inward bent with the radius of $50 \mathrm{~mm}$ as shown in the inset of Fig. 3(c). The diodes bared tensile and compressive stresses during outward and inward bending, respectively. The $J-V$ characteristics of the diodes are nearly consistent during outward and inward bending, and can recover to the initial state after several bending circles, as shown in Fig. 3(c) and (d), indicating that these diodes work well under the tensile and compressive stresses. To study the device uniformity, 10 diodes randomly selected on PET or PI substrates were tested, as shown in Fig. 3(e) and (f). and the results show that these flexible diodes have high uniformity.

\section{Reverse current mechanism of flexible diodes.}

In order to understand the improvements in $J_{\text {off }}$ and $n$ of the diodes after being kept in air for 12 and 25 months, the reverse current has been analyzed according to the image force lowering model and the barrier inhomogeneity model [36]-[38]. As shown in Fig. 5, the diodes showed non-ideal reverse characteristics, i.e., a flat reverse current equaling to $J_{\mathrm{s}}$ [30], especially for the diode on PI. Such a non-ideal reverse current is always seen in the reported oxide semiconductor-based Schottky diodes, but it is hardly discussed [10]-[12], [24], [26]. At the Schottky junction, the electrons in the IGZO and the induced charges on the Pd surface create an electrical field at the IGZO/Pd interface, resulting in a lower barrier height, and 

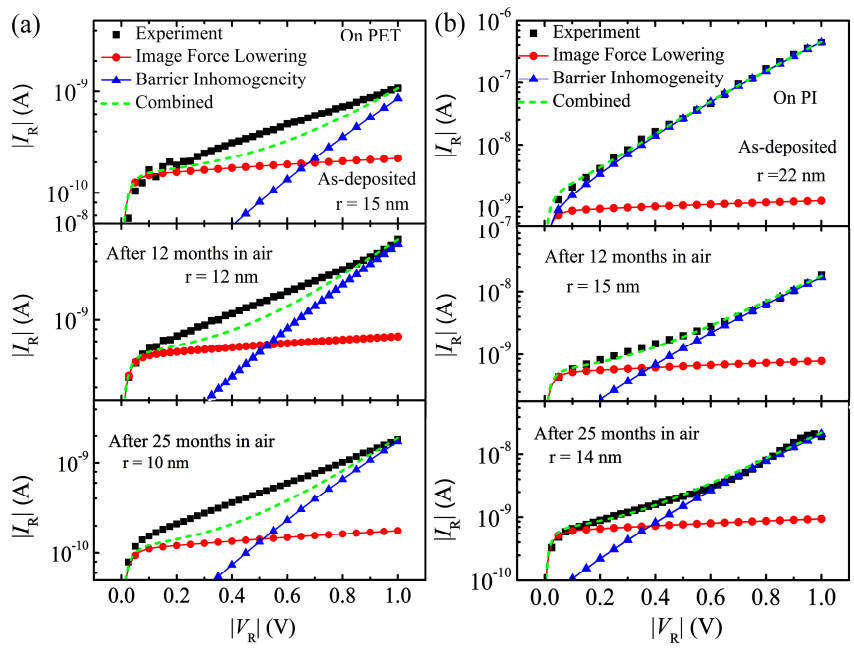

Fig. 5. $\left|I_{\mathrm{R}}\right|-\left|V_{\mathrm{R}}\right|$ characteristics for the as-deposited diodes and the same diodes kept in ambient air for 12 and 25 months on PET (a) and PI (b).

this is known as "image force lowering" [30]. At reverse biases, the applied voltage further lowers the barrier, which is described by [30],

$\left|I_{R}\right|=A A^{*} T^{2} \exp \left[-\frac{\Phi_{B}-\Delta \Phi}{k T}\right]\left[1-\exp \left(\frac{-q V_{R}}{n k T}\right)\right]$

$\Delta \Phi=q\left[\frac{q^{2} N_{d e p l}\left(\Phi_{B}-q V_{R}\right)}{8 \pi^{2}\left(\varepsilon_{s} \varepsilon_{0}\right)^{3}}\right]^{1 / 4}$

where $\left|I_{\mathrm{R}}\right|$ is the absolute reverse current, $V_{\mathrm{R}}$ is the reverse applied voltage, and $\Delta \Phi$ is the energy caused by image force lowering. In Fig. 5, the fittings based on image force lowering are in good agreement with the experimental results at low reverse biases. The obtained values of $\Phi_{\mathrm{B}}$ and $N_{\mathrm{depl}}$ are shown in Table II. However, the fittings show large deviation at high reverse biases, implying that the image force lowering is not the only factor affecting the reverse current.

Tung proposed that in addition to the image force lowering, Schottky barrier inhomogeneities also affect the reverse current [37]. Such model assumes that there are small patches with the barrier heights lower by value of $q \Delta \mathrm{V}$ than the mean barrier height, distributed at the Schottky interface [38]. The carrier conduction path prefers the patches with low barrier heights than high barrier height patches. Each low barrier region results in a saddle point in the conduction band of IGZO at some distance away from the Schottky interface, and it is suggested that the electron transport is mainly determined by the potential at such saddle point [37], [38]. The reverse biases can further lower the potential at the saddle points, resulting in a clearly increased $I_{\mathrm{R}}$ depending on $V_{\mathrm{R}}$ [37], [38]. By assuming a circular geometry of the patches, the leakage current caused by the barrier inhomogeneity is given by [37],

$I_{\text {patch }}=A_{\text {eff }} A^{*} T^{2} \exp \left[-\frac{\Phi_{\text {eff }}}{k T}\right]\left[1-\exp \left(\frac{-q V_{R}}{n k T}\right)\right]$

$A_{e f f}=\frac{4 k T \gamma}{9 q\left(\Phi_{B}-q V_{R}\right)^{2 / 3}}\left(\frac{\varepsilon_{s} \varepsilon_{0}}{N_{d e p l}}\right)^{2 / 3}$

$\Phi_{e f f}=\Phi_{B}-\gamma\left[\frac{N_{\text {depl }}\left(\Phi_{B}-q V_{R}\right)}{\varepsilon_{s} \varepsilon_{0}}\right]^{1 / 3}$

where $I_{\text {patch }}$ is the reverse current through the patch with a lower barrier height, $A_{\text {eff }}$ is the effective are of the patch, $\Phi_{\text {eff }}$ is the effective barrier height at reverse biases, and $\gamma$ is used to describe the barrier uniformity, given by $\gamma=3\left(\Delta \mathrm{V} r^{2} / 4\right)^{1 / 3}$, where $r$ is the effective radius of the patch. By using this model, $I_{\text {patch }}$ can be extracted from the difference of the experimental result and the calculated image force lowering current. In Fig. 5, the final fittings are in good agreement with the reverse currents. Fig. 5(a) shows the reverse current characteristics of the asdeposited diode on PET and the diode being kept in ambient air after 12 and 25 months. The fitting shows that $\gamma=1.46 \times 10^{-4}$, $1.28 \times 10^{-4}$, and $1.11 \times 10^{-4} \mathrm{~cm}^{2 / 3} \mathrm{~V}^{1 / 3}$ for the as-deposited diode, and the diode keeping in ambient air for 12 and 25 months, respectively. By assuming an appropriate $\Delta \mathrm{V}=0.2 \mathrm{~V}$ [38], it is found that the effective radius of the patch decreases from 15 to 12 and $10 \mathrm{~nm}$ after being kept in ambient air for 12 and 25 months, respectively. For the diode on PI, as shown in Fig. 5(b), the effective radius of the patch decreases from $22 \mathrm{~nm}$ for the as-deposited diode to 15 and $14 \mathrm{~nm}$ for the diode after keeping in ambient air for 12 and 25 months, respectively. These results indicate improved barrier uniformity by storage in ambient air for more than two years, which is in accordance with the improved $n$ from 1.22 and 1.19 to 1.09 and 1.11 for diodes on PET and PI, respectively. The improvement is most likely due to a slow filling up of oxygen vacancies in the IGZO film and partiality in the region close to the Schottky interface.

TABLE III

SUMMARY OF FLEXIBLE SCHOTTKY JUNCTIONS TO OXIDE SEMICONDUCTORS REPORTED IN THE LITERATURE.

\begin{tabular}{ccccc}
\hline \hline Substrate & Schottky junction & $\Phi_{\mathrm{B}}(\mathrm{eV})$ & $n$ & $I_{\text {on/off }}$ \\
\hline${ }^{\text {aPI }}($ ref.9) & IGZO/Pt & $\sim 0.8$ & $\sim 1.2$ & $\sim 3 \times 10^{5}( \pm 1 \mathrm{~V})$ \\
PET (ref.9) & IGZO/Pt & -- & -- & $\sim 10^{3}( \pm 1 \mathrm{~V})$ \\
PET (ref.10) & IGZO/Pt & 0.51 & 2.1 & $\sim 10^{3}( \pm 1 \mathrm{~V})$ \\
COC (ref.11) & IGZO/PEDOT:PSS & -- & $\sim 1.4$ & $\sim 5 \times 10^{5}( \pm 1 \mathrm{~V})$ \\
Paper (ref.12) & IGZO/Ru-Si-O & 0.79 & 2.13 & $8.4 \times 10^{5}( \pm 2 \mathrm{~V})$ \\
PEN (ref.13) & n-IGZO/P-Cu $2 \mathrm{O}$ & -- & 1.4 & $3.4 \times 10^{4}( \pm 1.2 \mathrm{~V})$ \\
PS(ref.14) & ZnO/Au & -- & 3.04 & $>10^{3}( \pm 3 \mathrm{~V})$ \\
PI (ref.39) & n-IGZO/P-NiO & -- & $\sim 3.2$ & $\sim 10^{4}( \pm 2.5 \mathrm{~V})$ \\
PET (ref. 40) & ZnO/Ag & 0.54 & 2.8 & $\sim 10^{2}( \pm 1 \mathrm{~V})$ \\
PET (our work) & IGZO/Pd & 0.80 & 1.09 & $2.0 \times 10^{7}( \pm 1 \mathrm{~V})$ \\
PI (our work) & IGZO/Pd & 0.78 & 1.11 & $1.3 \times 10^{6}( \pm 1 \mathrm{~V})$ \\
\hline \hline
\end{tabular}

Poly(3,4-ethylenedioxythiophene):poly(styrenesulfonate) (PEDOT:PSS), Cyclic olefin copolymer (COC), Polyethylene Naphthalate (PEN), Polystryene (PS). ${ }^{\mathrm{a}}$ The diodes were annealed in $\mathrm{O}_{2}(1 \mathrm{~atm})$ at $200^{\circ} \mathrm{C}$ for $1 \mathrm{~h}$

\section{The breakdown voltage}

These flexible diodes show relatively low reverse current density which is even improved after being kept in ambient air for more than two years. The low reverse current could result in a high reverse breakdown voltage, $V_{\mathrm{BR}}$. $V_{\mathrm{BR}}$ is a key parameter for Schottky diodes because that the diodes are usually subject to a much higher reverse bias than the forward voltage in many applications [31]. We have investigated the breakdown behavior of these flexible diodes under reverse biases as shown in Fig. 6(a). Ten diodes on PET, randomly selected in an area of $1.5 \mathrm{~cm} \times 1.5 \mathrm{~cm}$, show relatively high $V_{\mathrm{BR}}$ of $7.5 \pm 0.7 \mathrm{~V}$, while the diodes on PI show slightly lower $V_{\mathrm{BR}}$ of $4.5 \pm 0.3 \mathrm{~V}$, as shown in Fig. 6(a) and (b). The higher $V_{\mathrm{BR}}$ of diodes on PET than those on PI is originated from the better Schottky interface

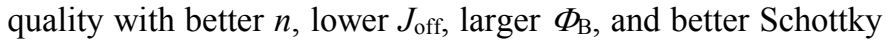


barrier homogeneity. It is worthy noted that the diodes on PET even have higher $V_{\mathrm{BR}}$ than those of diodes on rigid glass with the same IGZO thickness $(100 \mathrm{~nm}, 6.2 \pm 0.7 \mathrm{~V})$ [31], as shown in Fig. 6(a) and (b), and those of rigid diodes with the same IGZO thickness based on the reported empirical linear dependence of $V_{\mathrm{BR}}$ on IGZO thickness [24], [31]. Our results thus indicate that with the optimized treatment, the flexible IGZO/Pd Schottky contacts can reach ultrahigh quality comparable or even better than the rigid ones. These results are highly inspiring for the real applications of flexible diodes.

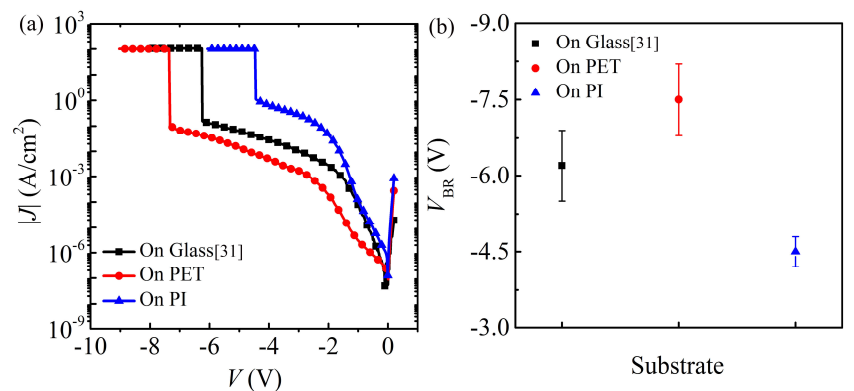

Fig. 6. (a) $J-V$ curves of the diodes on different substrates. (b) Breakdown voltage of the three diodes on different substrates. Experimental data on glass substrate are reproduced with permission [31].

\section{CONCLUSION}

In summary, based on the synergic optimization of Pd anode treatments and IGZO deposition, high performance flexible IGZO-based Schottky diodes have been achieved without any thermal treatment. The $J-V, C-V$, and XPS characterizations all indicated greater improved Schottky interface by using the oxygen plasma treatment than the UV-ozone treatment, due to a more completed Pd oxidation. These flexible diodes show high stability upon tensile and compressive stresses, comparable breakdown behavior as their rigid counterpart, and even improved performance after keeping in ambient air for up to 25 months due to improved barrier uniformity. The barrier height inhomogeneity of these flexible diodes has been evaluated by reverse current simulations, and the results explain well the aging performance improvement. The optimized flexible diodes on PET show very high performance with a near unity ideality factor of 1.09 , high barrier height of $0.8 \mathrm{eV}$, high rectification ratio of $2.0 \times 10^{7}$, and breakdown voltage of $\sim 7.5$ $\mathrm{V}$, and to the best of our knowledge, this is the best overall performance among the flexible diodes reported to date.

\section{REFERENCES}

[1] T. Someya, Y. Kato, T. Sekitani, S. Iba, Y. Noguchi, Y. Murase, T. Sakurai, "Conformable, flexible, large-area networks of pressure and thermal sensors with organic transistor active matrixes," Proc. Natl. Acad. Sci. USA., vol. 102, no. 35, pp. 12321-12325, Aug. 2005, DOI: 10.1073/pnas.0502392102.

[2] C. Pang, C. Lee, K. Y. Suh, "Recent Advances in Flexible Sensors for Wearable and Implantable Devices," J. Appl. Polym. Sci., vol. 130, no. 3, pp. 1429-1441, Jun. 2013, DOI: 10.1002/app.39461.

[3] R. Singh, E. Singh, H. S. Nalwa, "Inkjet printed nanomaterial based flexible radio frequency identification (RFID) tag sensors for the internet of nano things," RSC Adv., vol. 7, no. 77, pp. 48597-48630, Oct. 2017, DOI: 10.1039/c7ra07191d.

[4] H. Yabuta, M. Sano, K. Abe, T. Aiba, T. Den, H. Kumomi, H. Hosono, "High-mobility thin-film transistor with amorphous $\mathrm{InGaZnO}_{4}$ channel fabricated by room temperature rf-magnetron sputtering," Appl. Phys. Lett., vol. 89, no. 11, p. 112123, Sept. 2006, DOI: 10.1063/1.2353811.

[5] K. Nomura, H. Ohta, A. Takagi, T. Kamiya, M. Hirano, H. Hosono, "Room-temperature fabrication of transparent flexible thin-film transistors using amorphous oxide semiconductors," Nature, vol. 432, no. 7016, pp. 488-492, Nov. 2004, DOI:10.1038/nature03090.

[6] M. Lorenz, A. Lajn, H. V. Frenzel, H. Wenckstern, M. Grundmann, P. Barquinha, E. Fortunato, "Low-temperature processed Schottky-gated field-effect transistors based on amorphous gallium-indium-zinc-oxide thin films," Appl. Phys. Lett., vol. 97, no. 24, p. 243506, Dec. 2010, DOI: 10.1063/1.3525932.

[7] L. Petti, N. Münzenrieder, C. Vogt, H. Faber, L. Büthe, G. Cantarella, G. Tröster, "Metal oxide semiconductor thin-film transistors for flexible electronics," Appl. Phys. Rev., vol. 3, no. 2, p. 021303, Jun. 2016, DOI: 10.1063/1.4953034

[8] J. S. Park, T. W. Kim, D. Stryakhilev, J. S. Lee, S. G. An, Y. S. Pyo, H. $\mathrm{K}$. Chung, "Flexible full color organic light-emitting diode display on polyimide plastic substrate driven by amorphous indium gallium zinc oxide thin-film transistors," Appl. Phys. Lett., vol. 95, no. 1, p. 013503 , Jul. 2009, DOI: 10.1063/1.3159832.

[9] D. H. Lee, K. Nomura, T. Kamiya, H. Hosono, "Diffusion-Limited aIGZO/Pt Schottky Junction Fabricated at $200^{\circ} \mathrm{C}$ on a Flexible Substrate," IEEE Electron Device Lett., vol. 32, no. 12, pp. 1695-1697, Oct. 2011, DOI: 10.1109/LED.2011.2167123.

[10] J. Zhang, Y. Li, B. Zhang, H. Wang, Q. Xin, A. Song, "Flexible indiumgallium-zinc-oxide Schottky diode operating beyond $2.45 \mathrm{GHz}$," Nat. Commun., vol. 6, p. 7561, Jul. 2015, DOI: 10.1038/ncomms8561.

[11] C. H. Chang, C. J. Hsu, C. C. Wu, "Rectified Schottky diodes based on PEDOT:PSS/InGaZnO junctions," Org. Electron., vol. 48, pp. 35-40, Sept. 2017, DOI: 10.1016/j.orgel.2017.05.035.

[12] J. Kaczmarski, M. A. Borysiewicz, K. Piskorski, M. Wzorek, M. Kozubal, E. Kamińska, "Flexible IGZO Schottky diodes on paper," Semicond. Sci. Technol., vol. 33, no. 1, p. 015010, Dec. 2017, DOI: 10.1088/1361-6641/aa9acb.

[13] W. C. Chen, P. C. Hsu, C. W. Chien, K. M. Chang, C. J. Hsu, C. H. Chang, C. C. Wu, "Room-temperature-processed flexible n-InGaZnO/p$\mathrm{Cu}_{2} \mathrm{O}$ heterojunction diodes and high-frequency diode rectifiers," J. Phys. D: Appl. Phys., vol. 47, no. 36, p. 365101, Aug. 2014, DOI: 10.1088/0022-3727/47/36/365101.

[14] S. Lu, J. Qi, S. Liu, Z. Zhang, Z. Wang, P. Lin, Y. Zhang, "Piezotronic Interface Engineering on $\mathrm{ZnO} / \mathrm{Au}$-Based Schottky Junction for Enhanced Photoresponse of a Flexible Self-Powered UV Detector," ACS Appl. Mater. Inter., vol. 6, no. 16, pp. 14116-14122, Jul. 2014, DOI: 10.1021/am503442c.

[15] Y. Zhang, Z. Mei, S. Cui, H. Liang, Y. Liu, X. Du, "Flexible Transparent Field-Effect Diodes Fabricated at Low-Temperature with All-Oxide Materials," Adv. Electron. Mater., vol. 2, no. 5, pp. 100486-100490, Mar. 2016, DOI: 10.1002/aelm.201500486.

[16] J. Kaczmarski, A. Taube, M. A. Borysiewicz, M. Myśliwiec, K. Piskorski, K. Stiller, E. Kamińska, "Transparent Ru-Si-O/In-Ga-Zn-O MESFETs on Flexible Polymer Substrates," IEEE Trans. Electron Devices., vol. 65, no. 1, pp. 129-135, Feb. 2018, DOI: 10.1109/TED.2017.2658939.

[17] A. Chasin, V. Volskiy, M. Libois, K. Myny, M. Nag, M. Rockelé, P. Heremans, "An integrated a-IGZO UHF energy harvester for passive RFID tags" IEEE Trans. Electron Devices., vol. 61, no. 9, pp. 3289-3295. Jul. 2014, DOI: 10.1109/TED.2014.2340462

[18] D. Li, M. Chen, Q. Zong, Z. Zhang, "Floating-Gate Manipulated Graphene-Black Phosphorus Heterojunction for Nonvolatile Ambipolar Schottky Junction Memories, Memory Inverter Circuits, and Logic 
Rectifiers," Nano lett., vol. 17, no. 10, pp. 6353-6359, Sept. 2017, DOI: 10.1021/acs.nanolett.7b03140

[19] J. W. Lee, H. M. Kwon, M. H. Kim, S. R. Lee, Y. B. Kim, D. K. Choi, "Self-Rectifying Effect in Resistive Switching Memory Using Amorphous InGaZnO," J. Electron Mater., vol. 43, no. 5, pp. 1384-1388, May. 2014, DOI: 10.1007/s11664-014-3083-8

[20] A. Chasin, L. Zhang, A. Bhoolokam, M. Nag, S. Steudel, B. Govoreanu, P. Heremans, "High-Performance a-IGZO Thin Film Diode as Selector for Cross-Point Memory Application," IEEE Electron Device Lett., vol. 35, no. 6, pp. 642-644, Apr. 2014, DOI: 10.1109/LED.2014.2314704.

[21] A. Chasin, S. Steudel, F. Vanaverbeke, K. Myny, M. Nag, T. H. Ke, P. Heremans, "UHF IGZO Schottky Diode," IEDM IEEE Internat., 2012, pp. 12.4.1-12.4.4, DOI: 10.1109/IEDM.2012.6479030.

[22] A. Chasin, M. Nag, A. Bhoolokam, K. Myny, S. Steudel, S. Schols, P. Heremans, "Gigahertz Operation of a-IGZO Schottky Diodes," IEEE Trans. Electron Devices., vol. 60, no. 10, pp. 3407-3412, Oct. 2013, DOI: 10.1109/TED.2013.2275250.

[23] J. Zhang, L. Zhang, X. Ma, J. Wilson, J. Jin, L. Du, A. Song, "Lowfrequency noise properties in Pt-indium gallium zinc oxide Schottky diodes," Appl. Phys. Lett., vol. 107, no. 9, p. 093505, Sept. 2015, DOI: 10.1063/1.4930019.

[24] A. Chasin, S. Steudel, K. Myny, M. Nag, T. H. Ke, S. Schols, P. Heremans, "High-performance a-In-Ga-Zn-O Schottky diode with oxygen-treated metal contacts," Appl. Phys. Lett., vol. 101, no. 11, p. 113505, Sept. 2012, DOI: 10.1063/1.4752009.

[25] G. T. Dang, T. Kawaharamura, M. Furuta, M. W. Allen, "Metalsemiconductor field-effect transistors with In-Ga-Zn-O channel grown by nonvacuum-processed mist chemical vapor deposition," IEEE Electron Device Lett., vol. 36, no. 5, pp. 463-465, Mar. 2015, DOI: 10.1109/LED.2015.2412124.

[26] J. Zhang, Q. Xin, A. Song, "High Performance Schottky Diode Based on Indium-Gallium-Zinc Oxide," J. Vac. Sci. Technol., A, vol. 34, no. 4, Apr. 2016, DOI: $10.1116 / 1.4945102$

[27] Y. Son, J. Li, R. L. Peterson, "In Situ Chemical Modification of Schottky Barrier in Solution-Processed Zinc Tin Oxide Diode," ACS Appl. Mater. Inter., vol. 8, no. 36, pp. 23801-23809, Aug. 2016, DOI: 10.1021/acsami.6b05953.

[28] L. Du, H. Li, L. Yan, J. Zhang, Q. Xin, Q. Wang, A. Song, "Effects of substrate and anode metal annealing on InGaZnO Schottky diodes," Appl. Phys. Lett., vol. 110, no. 1, p. 011602, Jan. 2017, DOI: 10.1063/1.4973693.

[29] K. Ide, Y. Kikuchi, K. Nomura, M. Kimura, T. Kamiya, H. Hosono, "Effects of excess oxygen on operation characteristics of amorphous InGa-Zn-O thin-film transistors," Appl. Phys. Lett., vol. 99, no. 9, pp. 093507, Sept. 2011, DOI: 10.1063/1.3633100

[30] S. M. Sze and K. K. Ng, Physics of Semiconductor Devices, 3rd ed. New York: Wiley, 2007.

[31] Q. Xin, L. Yan, Y. Luo, A. Song, "Study of breakdown voltage of indium-gallium-zinc-oxide-based Schottky diode," Appl. Phys. Lett., vol. 106, no. 11, p. 113506, Mar. 2015, DOI: 10.1063/1.4916030.

[32] T. A. Beierlein, W. Brütting, H. Riel, E. I. Haskal, P. Müller, W. Rieß, "Kelvin probe investigations of metal work functions and correlation to device performance of organic light-emitting devices," Synth. Met., vols. 111-112, pp. 295-297, Jun. 2000, DOI: 10.1016/S0379-6779(99)004506.

[33] K. R. Peta, B. G. Park, S. T. Lee, M. D. Kim, J. E. Oh, T. G. Kim, V. R. Reddy, "Analysis of electrical properties and deep level defects in undoped GaN Schottky barrier diode," Thin solid films, vol. 534, pp. 603608. May. 2013, DOI: 10.1016/j.tsf.2013.01.100

[34] J. H. Evans-Freeman, M. M. El-Nahass, A. A. M. Farag, A. Elhaji, "Current transport mechanisms and deep level transient spectroscopy of Au/n-Si Schottky barrier diodes," Microelectron. Eng., vol. 88 no. 11, pp. 3353-3359, Nov. 2011, DOI: 10.1016/j.mee.2011.07.001
[35] D. K. Schroder, Semiconductor material and device characterization. John Wiley \& Sons, 2006.

[36] J. H. Werner, H. H. Güttler, "Barrier inhomogeneities at Schottky contacts," J. Appl. Phys., vol. 69, no. 3, pp. 1522-1533, Jun. 1991, DOI: $10.1063 / 1.347243$.

[37] R. T. Tung, "Electron transport at metal-semiconductor interfaces: General theory," Phys. Rev. B., vol. 45, no. 23, pp. 13509-13523, Jun. 1992, DOI: 10.1103/PhysRevB.45.13509.

[38] J. Wilson, J. Zhang, Y. Li, Y. Wang, Q. Xin, A. Song, "Influence of interface inhomogeneities in thin-film Schottky diodes," Appl. Phys. Lett., vol. 111, no. 21, p. 213503, Nov. 2017, DOI: 10.1063/1.5004247.

[39] N. Münzenrieder, C. Zysset, L. Petti, T. Kinkeldei, G. A. Salvatore, G. Tröster, "Room temperature fabricated flexible $\mathrm{NiO} / \mathrm{IGZO}$ pn diode under mechanical strain," Solid-State Electron., vol. 87, pp. 17-20, Sept. 2013, DOI: 10.1016/j.sse.2013.04.030.

[40] X. Zhang, J. Zhai, X. Yu, L. Ding, W. Zhang, "Fabrication and characterization of flexible $\mathrm{Ag} / \mathrm{ZnO}$ Schottky diodes on polyimide substrates," Thin Solid Films, vol. 548, pp. 623-626, Dec. 2013, DOI: 10.1016/j.tsf.2013.09.090

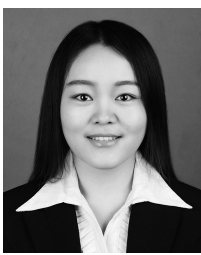

Lulu Du was born in Linyi City, Shandong Province, in 1990. She received the B. S. degree in physics from Shandong University of Technology, Zibo, in 2014, and is currently working toward the $\mathrm{Ph}$. D. degree in microelectronics and solid-state electronics at Shandong University, Jinan. Her research interests include rectifier diodes, thin film transistors and high power devices based on oxide semiconductor.

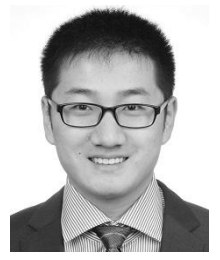

Jiawei Zhang received the M.S. degree in photo science and the $\mathrm{Ph}$. D. degree in electronics from the University of Manchester, Manchester, U.K., in 2012 and 2016, respectively. He has been a Research Assistant with the School of Electrical and Electronic Engineering, the University of Manchester,

since 2016.

His current research interests include the oxide semiconductors, thin-film transistors, high-frequency electronics, and 2-D materials.

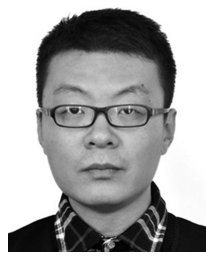

Yunpeng Li received the B.S. degree in microelectronics and solid-state electronics from Shandong University, Jinan, in 2014, where he is currently pursuing the Ph.D. degree in microelectronics and solid-state electronics.

His current research interests include fabrication and design of integrated circuits based on oxide TFTs.

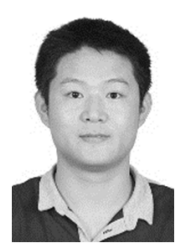

Mingsheng Xu received the B.S. in Physics, and $\mathrm{Ph}$. D. degrees in Materials Physics and Chemistry from Shandong University, Jian, China, in 2009 and 2014, respectively. From 2014 to 2017, he held a post-doctoral position in Electronics Science and Technology with South China University of Technology, Guangzhou, China. Since 
2017, he has been an associate professor with the school of Microelectronics, Shandong University.

His research interests include wide band-gap semiconductor materials growth and devices fabrication.

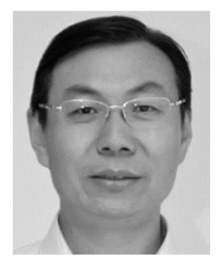

Qingpu Wang was born in September 1963. He received the B.S. and M.S. degrees in physics, in 1987 and 1998, respectively, and the Ph.D. degree in condensed matter physics from Shandong University (SDU), Jinan, China, in 2003. From 2013 to 2016, he was with the School of Information Science and Engineering, SDU. Since 1987, he has been with the Department of Physics, SDU.

Dr. Wang is a member of the Chinese Physical Society and Shandong Physical Society. He is the Reader of Journal of Semiconductor, Chinese Physics Letter, Journal of Chinese Physics B, etc.

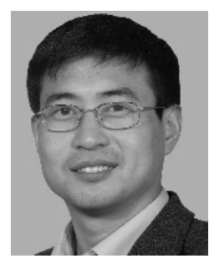

Aimin Song (M'02-SM'07) received Ph.D. degree from the Institute of Semiconductors, Chinese Academy of Sciences in 1995. After three years of fellowships at University of Glasgow and University of Munich by the Royal Society and Alexander von Humboldt Foundation, he was a Guest Lecturer at Lund University before moving to Manchester as a Lecturer in 2002. He became a Professor of nanoelectronics in 2006.

His research is focused on novel nanoelectronic devices and thin-film electronics. Dr. Song's work has led to awards including "Researcher of the Year" Distinguished Achievement Medal of the University of Manchester, Royal Society Brian Mercer Feasibility Award, and a number of best conference paper awards.

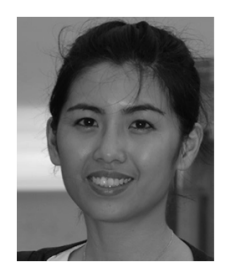

Qian Xin was born in Shandong, China, in 1982. She received the B.S. and Ph. D. degrees in material science from Shandong University, Jinan, China, in 2003 and 2008, respectively. From 2009 to 2012, she held a post-doctoral position in surface and interface science with Chiba University, Chiba, Japan. Since 2012, she has been an Associate Professor with the School of Microelectronics, Shandong University.

Her research interests include metal oxide semiconductors, flexible electronics and displays, and surface and interface electronic structure studies by photoemission spectroscopy. 